\title{
Periventricular Leukomalacia
}

National Cancer Institute

\section{Source}

National Cancer Institute. Periventricular Leukomalacia. NCI Thesaurus. Code C99013.

Necrosis and softening of the white matter around the ventricles in the brain. It is usually seen in preterm infants and caused by lack of blood flow and oxyg enation in the periventricular brain parenchyma. 